\title{
Edeldisteln, beliebte Insektennahrungspflanzen für den Garten
}

\author{
Hilke Steinecke \& Marco Schmidt
}

\begin{abstract}
Eryngoes (Eryngium) are well suited for a natural garden in sunny, dry locations. They are a favourite nectar and pollen plant for many insects. Some species for the garden, especially Eryngium giganteum, and their pollinators are briefly presented.
\end{abstract}

\section{Zusammenfassung}

Edeldisteln (Eryngium) eignen sich gut für einen Naturgarten an sonnig-trockenen Standorten. Sie sind für viele Insekten eine beliebte Nektar- und Pollenpflanze. Einige Arten für den Garten, vor allem Eryngium giganteum, und ihre Bestäuber werden kurz vorgestellt.

\section{Insekten brauchen Futterpflanzen im Garten}

Wer Insektenvielfalt in seinem Garten fördern will, muss unbedingt auch darauf achten, geeignete Futterpflanzen für die ausgewachsenen Insekten sowie deren Larven anzusiedeln. Idealerweise werden heimische Pflanzenarten bevorzugt, weil heimische Insektenarten oft ganz spezielle Ansprüche an ihre Futterpflanze haben; und das sind dann natürlich auch heimische Pflanzenarten. Von Zierpflanzen mit gefüllten Blüten oder Gartenhybriden ist abzusehen, da diese oft steril sind, weder Nektar noch Pollen produzieren und für die Insekten „leere“ Mogelpackungen sind. Es gibt aber trotzdem eine Reihe von Wildpflanzen aus anderen Regionen der Erde, die sehr gut als Futterpflanzen angenommen werden, dazu pflegeleicht und attraktiv sind und deshalb für einen Naturgarten sehr geeignet sind.

\section{Insekten „stehen auf“ Edeldisteln}

Solche „Insekten-Lieblinge“ sind verschiedene Arten der Edeldistel, die auch Mannstreu genannt werden. Sie gehören der über 200 Arten umfassenden Gattung Eryngium an. Von ihnen gibt es mehrjährige und einjährige Arten. Obwohl die Edeldistel feste, stechende Blätter hat und auch ihre Blütenstände an eine Distel erinnern, ist Eryngium kein Korbblütler wie die Disteln. Die Gattung wird zu den Doldenblütlern (Apiaceae) gestellt. Der Blütenstand hat aber nicht die in dieser Familie weit verbreitete Form einer Doppeldolde. Eryngium bildet einfache Dolden mit sehr kurzen Blütenstielen der einzelnen Blüten.
Dadurch wird der gestauchte Blütenstand köpfchen- bis walzenförmig. Die Köpfchen werden am Grund von festen, stechenden Hochblättern umgeben, so dass der Eindruck einer großen Einzelblüte entsteht. Blüten und Hochblätter sind oft silbrigblau bis grünlich gefärbt.

Eine bekannte Art ist die an europäischen Küsten in Dünen wachsende Stranddistel (Eryngium maritimum). Sie wird $60 \mathrm{~cm}$ hoch mit weißlich bereiften Stielen. Die halbkugeligen Köpfchen werden von relativ breiten, sich überlappenden, graublauen Hüllblättern umgeben.

Bei uns auf Kalk-Magerrasen anzutreffen ist die Feld-Mannstreu (Eryngium campestre). Der Blütenstand ist in seiner oberen Hälfte sparrig verzweigt. Die kugeligen Köpfchen werden von schmalen graugrünen, weit voneinander $a b-$ stehenden Hüllblättern umgeben. Durch ihre blaue Blütenfarbe besonders attraktiv ist die Flachblatt-Mannstreu (Eryngium planum) aus Mittel-, Südost- und Osteuropa. Die unteren Blätter sind ungeteilt, die oberen 3-5-spaltig. Der Blütenstand ist eiförmig, die schmalen Hüllblätter sind relativ klein und überlappen sich nicht.

Ihrem Namen Amethyst-Mannstreu macht Eryngium amethystinum mit ihren intensiv violettbis metallisch blau gefärbten Blütenständen alle Ehre. Die fein eingeschnittenen Hüllblätter sind silbriggrau. Die Art kommt von Nord-Italien bis in den Nahen Osten vor. Ebenso eine attraktive Zierpflanze für unsere Gärten ist mit $40 \mathrm{~cm}$ Höhe die 


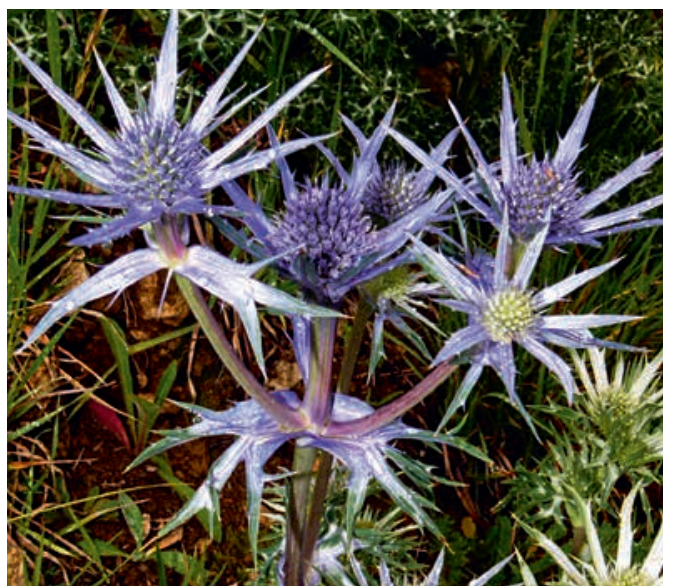

Abb. 1: Eryngium bourgatii mit blauen, distelartigen Köpfchen. (Foto: H. STEinecke)

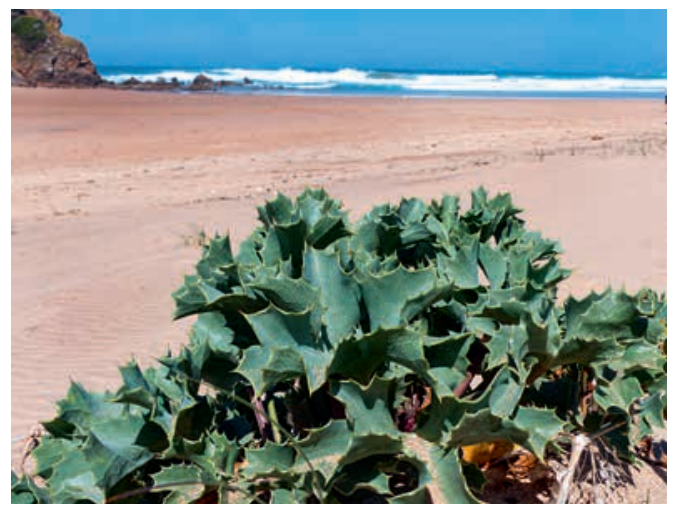

Abb. 3: Habitus von Eryngium maritimum mit blaugrauen, stacheligen Blättern. (Foto: H. Steinecke)

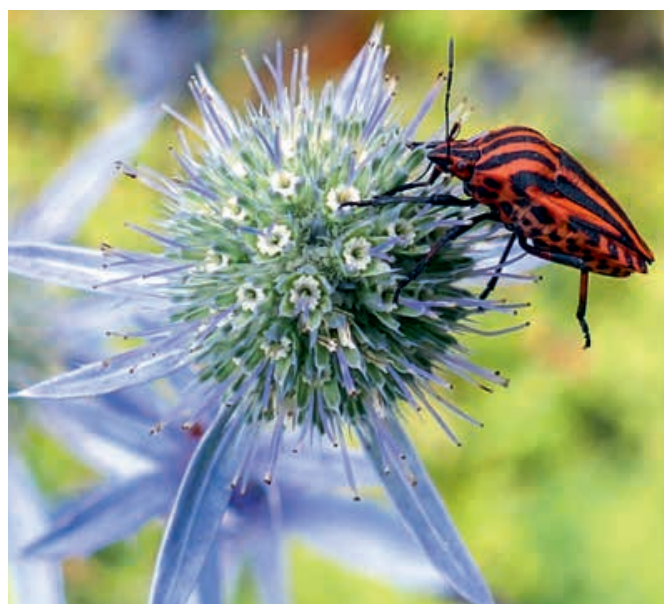

Abb. 5: Köpfchen von Eryngium planum mit Europäischer Streifenwanze (Graphosoma italicum). (Foto: H. STEInecke)

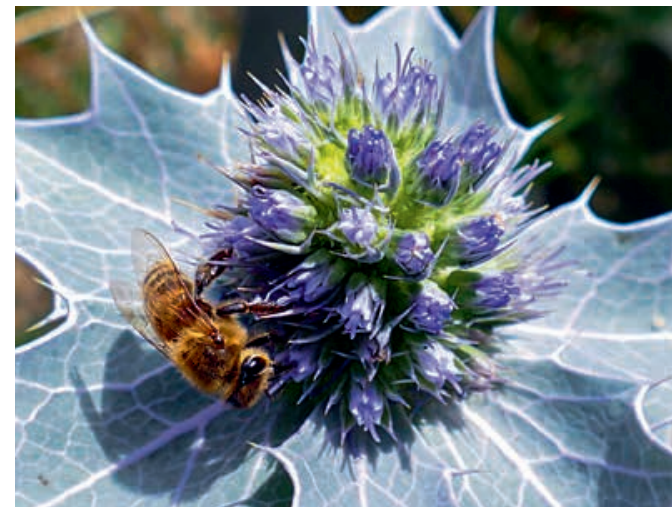

Abb. 2: Eryngium maritmum mit Besuch einer Honigbiene. (Foto: H. STEINecke)

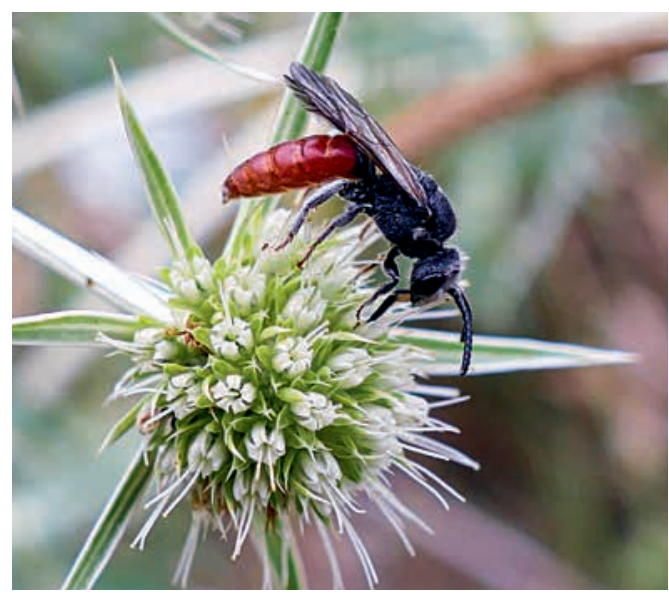

Abb.4: Köpfchen von Eryngium campestre mit Riesen-Blutbiene (Sphecodes albilabris). (Foto: H. Steinecke)

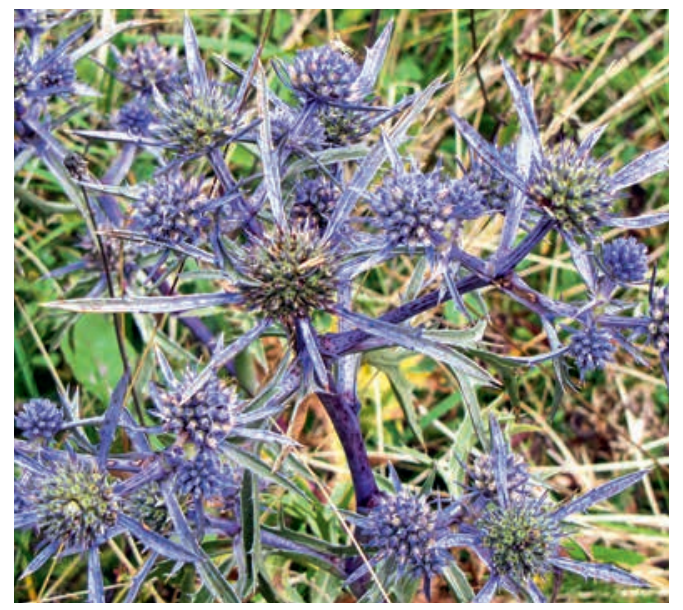

Abb. 6: Eryngium amethystinum. (Foto: H. STEINECKE) 


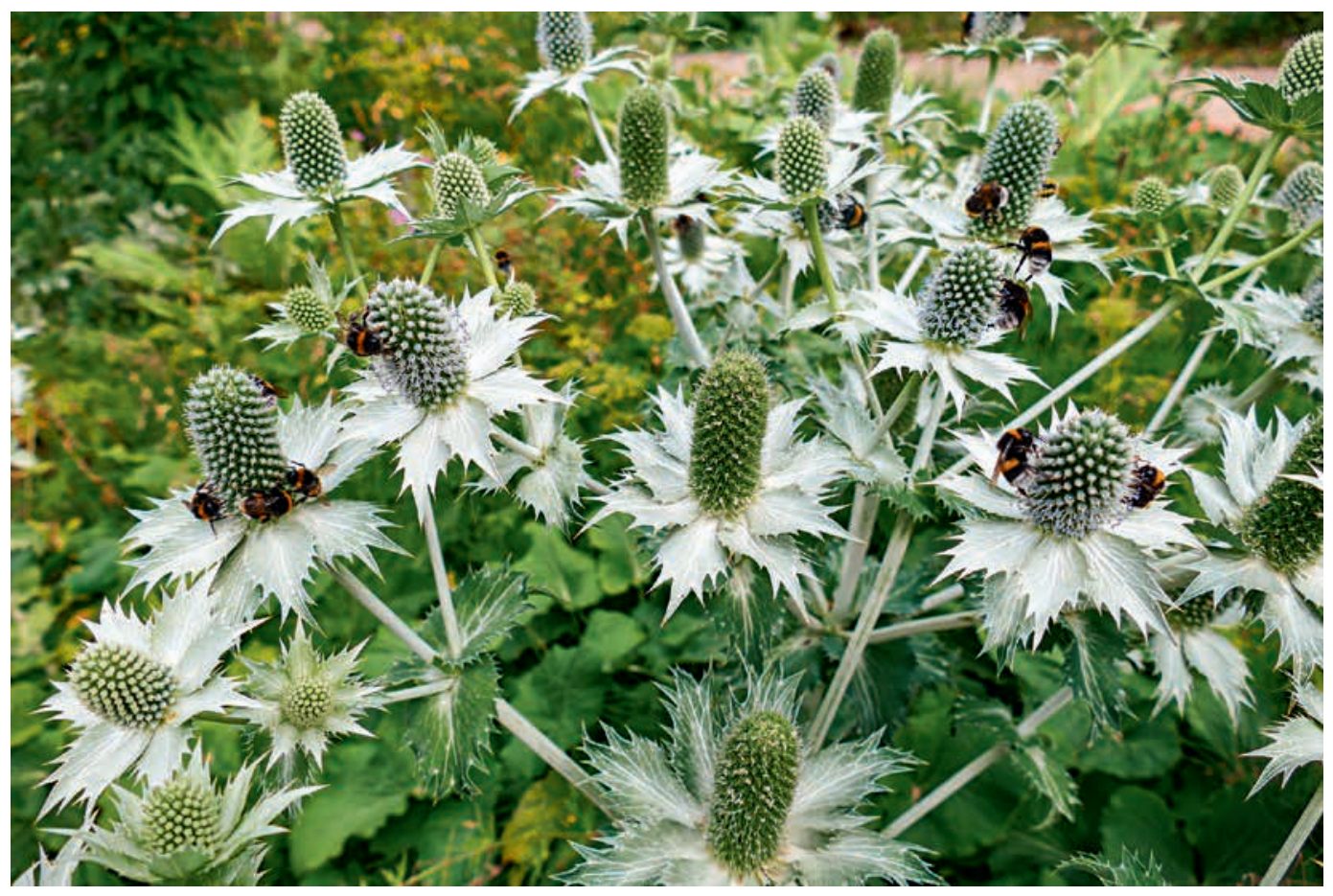

Abb. 7: Eryngium giganteum mit regem Hummelbesuch. (Foto: H. STEInecke)

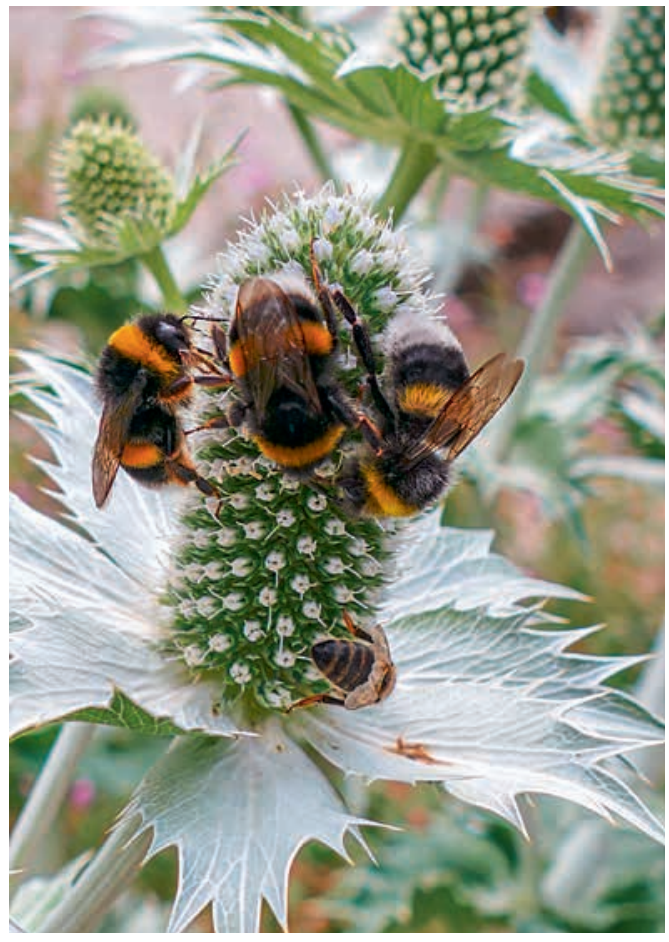

Abb. 8: Elfenbeindistel: Wo Hummeln sich tummeln. (Foto: H. STEINECKe)

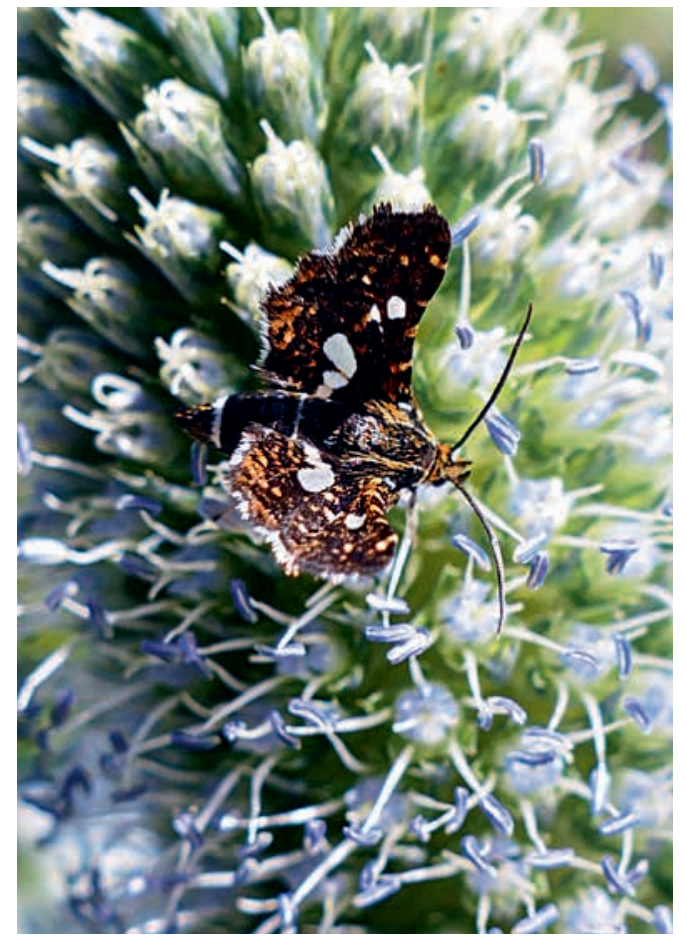

Abb. 9: Thyris fenestrella auf Eryngium giganteum.

(Foto: H. Steinecke) 


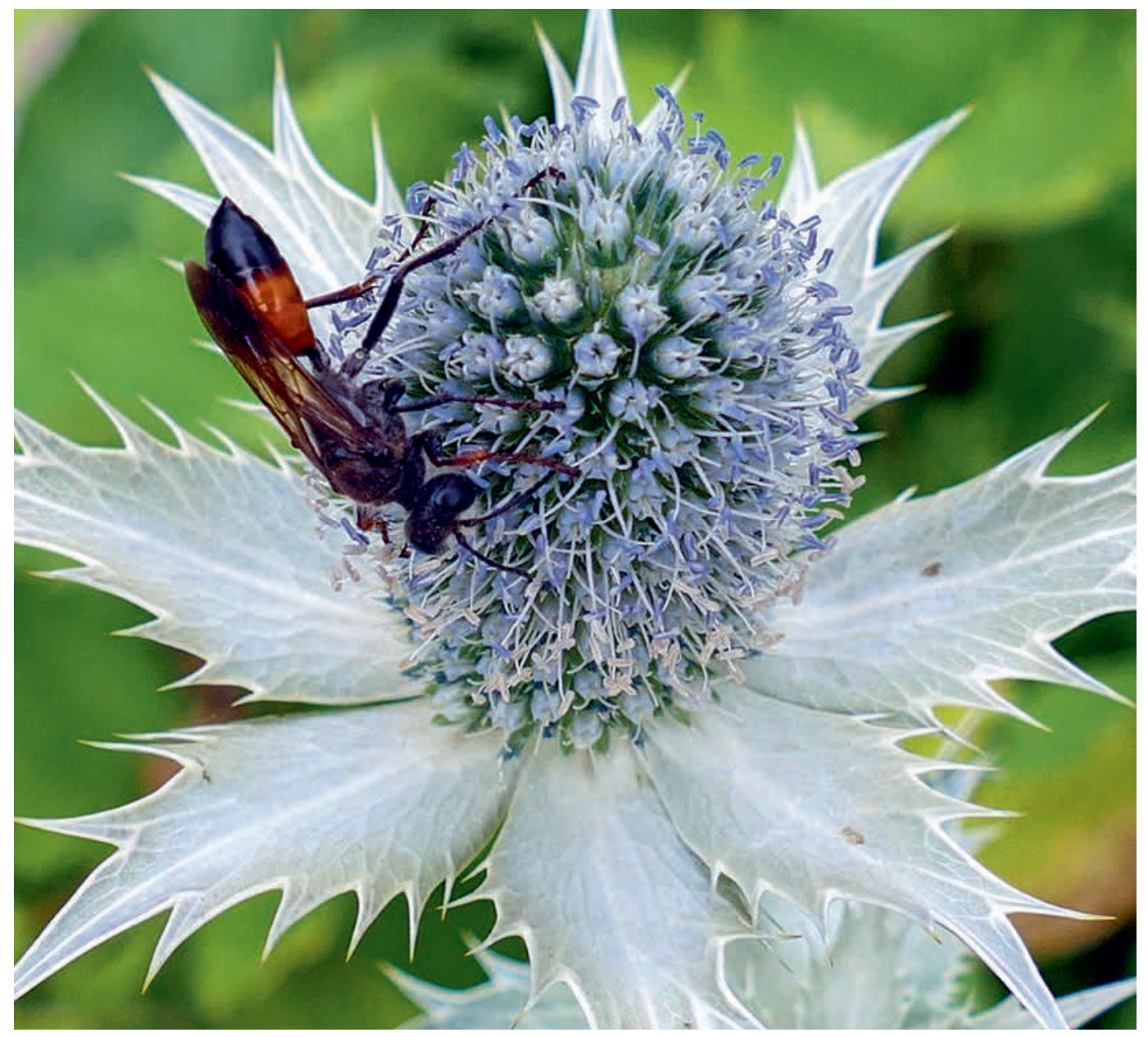

Abb. 10: Heuschreckensandwespe (Sphex funerarius) auf Eryngium giganteum. (Foto: H. STEINecke)

relativ kleine, in West-Europa und Nord-Afrika heimische Spanische Edeldistel (Eryngium bourgatii). Die hellblauen kugeligen Köpfchen werden von stahlblauen schmalen Hüllblättern umgeben.

\section{Miss Willmott's Ghost}

Vielleicht die auffälligste Art ist die einen knappen Meter hohe Elfenbeindistel (Eryngium giganteum). Sie wurde um 1800 aus dem Kaukasus und dem Iran eingeführt. Erstmals sicher nachgewiesen wurde sie 1824 im Garten des Apothekers JoHAnN Nikolaus Buek in Frankfurt/Oder. Die Art versamt sich leicht, in England ist sie als Neophyt weit verbreitet. Dort bezeichnet man sie als Miss Willmott's Ghost, weil die gleichnamige begeisterte Gärtnerin Ellen Willmott (1858-1934) bei
Gartenbesuchen wohl heimlich Samen verstreute, aus denen dann wie von Geisterhand überall Pflanzen entstanden. Eryngium giganteum zieht diverse Insekten stark an. Die Blütezeit reicht bis in den August, die alten Blütenstände eignen sich als Trockenblumen.

\section{Insektenvielfalt an den Blüten}

Alle genannten Arten eignen sich für sonnige Standorte auf wasserdurchlässigem Boden, sind deshalb auch dankbare Pflanzen in zunehmend heißeren und trockeneren Sommern. Edeldisteln üben eine starke Anziehungskraft auf diverse Insekten aus. Da der Nektar am Fruchtknoten der kleinen flachen Blüten gebildet wird, ist dieser auch für Insekten mit kurzen Mundwerkzeugen 


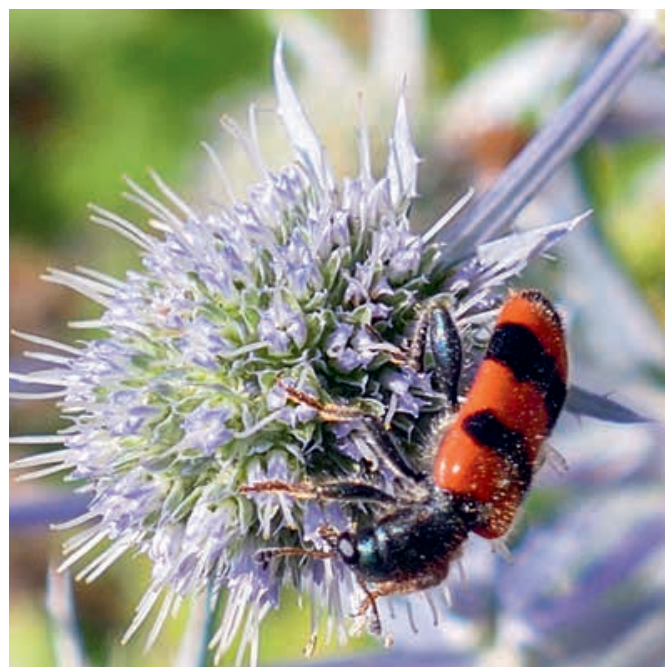

Abb. 11: Trichodes apiarius auf Eryngium planum.

(Foto: H. STEINecke)

leicht erreichbar. Insekten verschiedener Ordnungen wie Hautfügler, Fliegen, Schmetterlinge oder Käfer sind auf den Blütenköpfchen regelmäßige Gäste. Letztere sind vor allem an Pollen als Futter interessiert. Auch Wanzen sind hier zu finden. Besonders die Europäische Streifenwanze (Graphosoma italica) ist häufig zu sehen, ihr rot-schwarz gestreifter Körper bildet einen attraktiven Kontrast zu den blauen Blütenständen. Die Wanzen nehmen Nektar auf, sie saugen aber auch den Pflanzensaft und paaren sich oft sogar auf den Blüten.

Wer sich Edeldisteln in den Garten holt und in die Nähe einer „Insektenpflanzung“ setzt, kann sich anhand des regen Blütenbesuches einen groben Überblick verschaffen, welche Hummeln und andere blütenbesuchenden Insekten sich generell in der näheren Umgebung tummeln.

In Beobachtungen auf der Bürgerwissenschaftsplattform iNaturalist (https://www.inaturalist. org/projects/tiere-und-pilze-in-frankfurtsbotanischen-garten) wurden bisher folgende Insekten auf Eryngium-Arten fotografisch dokumentiert, was natürlich nicht bedeutet, dass nur diese Insekten die Blüten aufsuchen.

Hautflügler: Andrena (Sandbienen), Apis mellifera (Honigbiene), Bombus (Hummeln), Cerceris

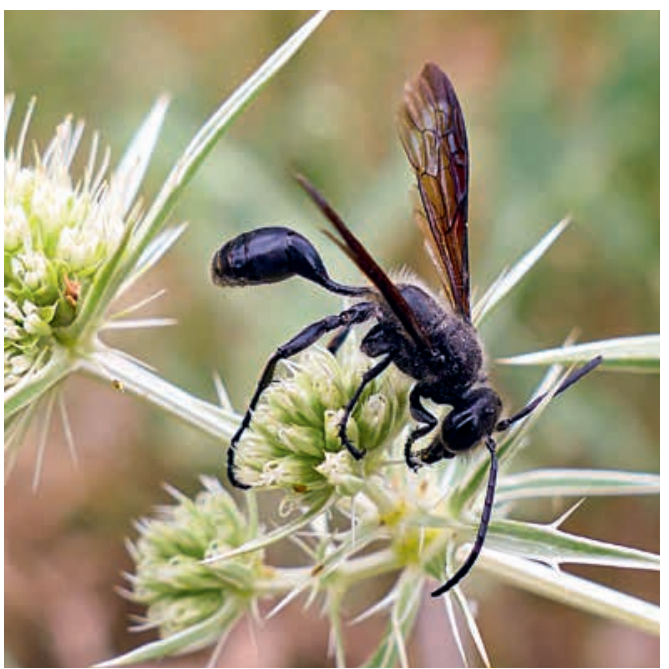

Abb. 12: Isodontia mexicana auf Eryngium campestre. (Foto: H. STEINECKe)

(Knotenwespe), Hylaeus (Maskenbienen), Isodontia mexicana (Mexikanischer Grillenjäger), Philanthus triangulum (Bienenwolf), Sphecodes (Blutbienen), Sphecodes albilabris (Riesen-Blutbiene), Sphex funerarius (Heuschreckensandwespe).

Fliegen: Myathropa florea (Totenkopf-Schwebfliege)

Schmetterlinge: Thyris fenestrella (WaldrebenFensterfleckchen)

Käfer: Trichius gallicus (Glattschieniger Pinselkäfer), Trichodes apiarius (Gemeiner Bienenkäfer)

Schnabelkerfe: Corizus hyoscyami (Zimtwanze), Graphosoma italicum (Europäische Streifenwanze)

\section{Anschriften der Autorin und des Autors}

Dr. Hilke Steinecke, Palmengarten Frankfurt, Siesmayerstr. 61, 60323 Frankfurt, E-Mail: hilke.steinecke@stadt-frankfurt.de; https://orcid.org/0000-0002-4618-4867

Dr. Marco Schмidt, Palmengarten Frankfurt, Siesmayerstr. 61, 60323 Frankfurt,

E-Mail: marco.schmidt@stadt-frankfurt.de; https://orcid.org/0000-0001-6087-6117 